\title{
L'aménagement intégré d'un grand fleuve : Le Rhône B. Evolution des équipements électromécaniques
}

\author{
Integrated development of a large river: the Rhone \\ B. Development of electro-mechanical equipments
}

\author{
P. Cazenave \\ Directeur de l'Exploitation \\ et des Services Electromécaniques \\ à la Compagnie Nationale du Rhône
}

L'équipement complet des usines, barrages et écluses du Rhône, entre les premières études de conception de Génissiat et la fin prochaine du dernier aménagement à réaliser (Loyettes) se sera étalé sur un demi-siècle. Il est naturel et heureux qu'il ait largement évolué. Cependant, cette évolution a toujours obéi à un certain nombre d'objectifs, agissant souvent simultanément, dont les principaux ont été :

- l'optimisation économique ;

- la sécurité de fonctionnement ;

- l'allègement de l'entretien;

- la souplesse de fonctionnement.

Si l'on met à part le cas particulier de Génissiat, chute moyenne $(70 \mathrm{~m})$ équipée de turbines Francis, dont nous ne détaillerons pas un équipement largement connu [1], qui a fait la preuve d'une fiabilité remarquable et qui reste, malgré son âge, tout à fait digne des conceptions actuelles, la totalité des aménagements est constituée de basses chutes dont la hauteur nominale varie entre $5,70 \mathrm{~m}$ (Vaugris) et 21 mètres (DonzèreMondragon).

Presque toutes ces chutes, équipées en aménagement mixte, avec écluse à grand gabarit, doivent respecter des contraintes d'exploitation liées à la production d'énergie et à la navigation. Elles doivent, en outre, s'accommoder :

- de variations importantes de débit (rapport de l'ordre de 5 à 8 entre le débit d'équipement et le débit d'étiage) - de variations corrélatives de chutes (généralement de $20 \%$, parfois de $50 \%$ entre étiage et débit d'équipement), ces variations étant souvent plus sensibles entre débit d'équipement et débit de crue annuelle.

Tous ces aménagements conçus suivant la technique des turbines axiales de basse chute

- soit verticales (pour les plus anciennes),

- soit horizontales (pour les plus récentes), ont inspiré une certaine homogénéîté des équipements qui s'est transformée en "paliers techniques" respectant d'une part, les quatre objectifs précités et d'autre part, les impératifs suivant :

- ajustement des données économiques,

- évolutions technologiques,

- expérience antérieures de conception et d'exploitation.

Les innovations, lorsqu'elles étaient importantes, ont toujours été introduites de façon à ne les généraliser qu'après une expérience suffisamment éprouvée selon nos propres conditions d'exploitation et avec toute l'exigence de fiabilité imposée :

- par les très faibles possibilités d'arrêt des groupes turbo-alternateurs (plus de 6000 heures de fonctionnement à pleine charge, c'est-à-dire plus de 7000 heures de rotation annuelle);

- par le fonctionnement permanent d'une voie navigable, jour et nuit, dimanches et fêtes compris avec seulement chaque année, 4 ou 5 jours consécutifs de "chômage technique", programmés plusieurs mois à l'avance pour assurer l'entretien des installations de cette voie navigable.

Nous examinerons, en centrant principalement l'exposé sur les aspects les plus hydrotechniques, la façon dont ces principes généraux se sont appliqués aux équipements des quatre principaux types d'ouvrages rencontrés sur ces aménagements mixtes à savoir : les usines, les déchargeurs, les écluses et les barrages.

\section{Usines et déchargeurs}

Nous traiterons de ces deux ouvrages simultanément, non seulement parce qu'ils constituent toujours un "bloc" de génie-civil commun, mais aussi parce que leur évolution hydraulique est étroitement imbriquée. 


\section{Turbines Kaplan}

Les premières usines de basse chute ont naturellement été équipées de turbines Kaplan, très classiques, de gros débit, de dimensions parmi les plus grandes pour l'époque de leur construction, l'optimisation ayant généralement conduit à des tracés assez rapides. En dehors de nombreuses améliorations technologiques ou auxiliaires apportées d'usine en usine, on doit noter au cours du temps les principales évolutions suivantes : - tendance à l'augmentation du débit d'équipement : à Bollène, le débit théorique d'équipement $\left(1530 \mathrm{~m}^{3} \mathrm{~s}\right)$ avait, sur la courbe des débits classés, une fréquence de 120 jours; à Bourg-lès-Valence, la fréquence du point correspondant $\left(2100 \mathrm{~m}^{3} / \mathrm{s}\right)$ était de 60 jours. Cela tient principalement à la croissance importante du coût de l'énergie prise en compte dans l'optimisation marginale ;

- tendance à élargir les possibilités de turbinage aux basses chutes, en permettant une surouverture en dessous de la chute nominale pour exploiter les turbines jusqu'aux limites de la cavitation ;

- "normalisation" des trois dernières chutes, aux mêmes dimensions principales ;

- tendance à remplacer la coupure de sécurité par déplacement de la vanne de l'amont vers l'aval.

\section{Vannes de sécurité}

Le dernier point signalé, cité à propos des turbines parce que le choix de l'équipement de sécurité est d'abord conditionné par le fonctionnement des turbines pendant leur coupure, mérite quelque développement, l'histoire du Rhône ayant eu un rôle important dans l'étude des vannes de coupure des groupes de basse chute. Au niveau d'un si bref exposé, on ne peut que se limiter aux jalons suivants :

a) Avant les conceptions de Seyssel et de DonzèreMondragon, une enquête internationale avait montré l'intérêt que présente l'installation d'une vanne de sécurité sur les usines de basse chute, les incidents d'emballement dûs à un mauvais fonctionnement des distributeurs (blocage, manque d'huile, grippage) étant d'une probabilité non négligeable, et les conséquences sur la machine, sur l'ouvrage et sur la sécurité générale, pouvant être très lourdes.

L'installation d'une vanne de sécurité a donc été adoptée généralement en France (et pas seulement sur le Rhône). Les techniques employées au début variaient avec les installations. Cependant, divers incidents constatés dans certaines usines françaises ou étrangères (cavitation en masse derrière des vannes par éléments empilés empêchant la descente des der. niers éléments, comportement brutal des turbines pendant les coupures...) avaient attiré l'attention sur les difficultés des coupures de débits des turbines par l'amont, et l'intérêt hydraulique des coupures par l'aval. C'est à Seyssel précisément, où la conception se prêtait bien à ce type d'essai, que la comparaison en vraie grandeur des coupures par l'amont et par l'aval fut effectuée. Elle concluait sans conteste à la supériorité hydraulique de la coupure par l'aval, qui fut retenue à Seyssel $(1950) *$. b) Cependant l'usine de Bollène (aménagement de Donzère-Mondragon) déjà très avancée à l'époque, était équipée, selon la tradition, d'une coupure par l'amont; d'autant que la coupure par l'aval se heurtait à des inquiétudes techniques (surpressions) et à des critiques d'objectif (protection de l'ensemble de l'usine, et non de la seule turbine). Cependant, en raison du coût de ces équipements de coupure à grande hauteur, forte charge, grand nombre de pertuis ( 3 pertuis d'entrée par turbine), on s'est orienté vers une vanne banale, appelée automatiquement sur le groupe en défaut. En l'absence d'une nouvelle analyse suffisamment avancée à l'époque de l'aménagement de Montélimar, une technique analogue a été adoptée à l'usine de Châteauneuf du Rhône (1953)*

c) Lors de l'équipement du Logis-Neuf, l'étude économique a montré que l'équipement banal mentionné ci-dessus se révélait nettement moins coûteux placé à l'aval que disposé à l'amont (pertuis moins nombreux et moins profonds). L'étude du génie civil montrait que, moyennant des lois de fonctionnement faciles à réaliser avec sûreté, les surpressions aval étaient aisément maintenues dans des limites qui n'influaient pas sur la conception ni de la machine, ni des ouvrages. On adopta donc une vanne aval banale (1956)*.

d) Lors de l'équipement de Beauchastel (1958)*, une étude plus intégrée entre constructeur de vannes et bureau de génie civil, tenant compte de tous les éléments auxiliaires (notamment de l'utilisation possible de la plateforme aval pour placer le poste de transformation) montrait que, pour une usine à 6 groupes $^{(1)}$, l'équipement en vannes individuelles en aval n'était pas plus cher qu'une solution avec vanne banale. On adopta donc cette solution, qui fut ensuite généralisée à tous les aménagements ultérieurs.

e) Une dernière évolution devait cependant achever l'œuvre : elle est due cette fois à la recherche d'un meilleur établissement de la marche en déchargeur, objet $\mathrm{du}$ chapitre suivant. Au lieu de vannes à manœuvre lente (environ $1 \mathrm{~m} / \mathrm{min}$ ), on conçut des vannes à descente rapide dans la première moitié de la course (environ $20 \mathrm{~m} / \mathrm{min}$ ) suivie d'un réglage en vitesse lente (environ $1 \mathrm{~m} / \mathrm{min}$ ). On abandonna alors la solution de Beauchastel (vannes flottantes équilibrées avec servomoteurs à double effet) pour des vannes à descente gravitaire et servomoteurs à simple effet, ces servomoteurs étant doublés pour éviter tout risque de chute libre et de surpression en résultant.

Cette solution a été absolument reconduite, avec seulement quelques variantes technologiques et dimensionnelles, depuis Pierre-Bénite (1962)*, y compris pour l'usine de Bourg-lès-Valence, dernière usine réalisée avec des groupes Kaplan.

f) A partir de l'étape précédente, les schémas de commande des vannes sont étroitement intégrés à ceux des turbines, pour réaliser avec certitude les séquences combinées nécessaires entre distributeur, pales et vannes,

* Les dates citées indiquent l'année du choix de conception.

(1) Il n'en est évidemment plus ainsi pour une usine à très grand nombre de groupes. Suivant les conceptions, "l'égalité" se situe aux environs de 6 à 8 groupes. 
cet ensemble devennant ainsi un véritable organe de contrôle du groupe, au même degré que distributeur et pales. Cette intégration apparut bientôt extrêmement intéressante pour une utilisation systématique de la vanne sur toute coupure de débit, cette méthode assurant, surtout sur les machines "très rapides", une coupure beaucoup plus calme que la traditionnelle coupure par le seul distributeur. Il est bien évident que ce rôle d'organe normal se trouvait ainsi tout préparé lorsque, pour des raisons d'économie, s'est introduite la technique des groupes simplifiés à "distributeurs et pales fixes" adoptés pour l'usine de Sauveterre (1969)*, de Caderousse (1971)* et de Vaugris (1976)*.

g) A noter également, pour clore ce chapitre sur les vannes, que la position des vannes dans le conduit des groupes bulbes a fait l'objet d'une étude d'optimisation, à partir de mesures de pertes sur modèle, pour rechercher la meilleure économie compte tenu du coût de la vanne (croissant avec la distance à la roue) et des pertes de rainure (décroissant avec cette distance).

On voit, à travers l'histoire de cet équipement, volontairement développé à titre d'exemple, comment, à l'occasion d'une chaîne d'aménagements réalisés avec des objectifs économiques et techniques de plus en plus affinés, s'imbriquent les traditionnelles notions de "fonction" et "d'organe" et comment réagissent les unes sur les autres les évolutions d'objectifs et d'équipement différents.

\section{Marche en déchargeur et ouvrage déchargeur}

Simultanément à toutes les études précédentes se développait, depuis Châteauneuf-du-Rhône, l'étude de la marche en déchargeur des turbines, qui a fait l'objet d'une session complète de la SHF [2].

Comme on le sait, toute usine hydroélectrique sur dérivation doit être associée au plus près des turbines, à un système de décharge du débit, permettant, en cas de déclenchement du réseau,

- d'une part, de minimiser les "ondes de disjonction" liées à la réduction du débit turbiné,

- d'autre part, de faciliter, en cas de panne longue, le basculement du débit de l'usine vers le barrage.

L'usine de Bollène (aménagement de DonzèreMondragon) avait été flanquée d'un ouvrage déchargeur très important, à vannes lentes, capable de rétablir la totalité du débit d'équipement. Les études sur les ondes de déclenchement [3] ont montré que si un tel équipement était idéal pour éviter le basculement du débit entre usine et barrage (ce qui serait très difficile à Bollène où la dérivation est de $30 \mathrm{~km}$ ), il était impuissant à lutter contre les ondes créés lors du déclenchement. C'est ce qui a conduit dans les aménagements ultérieurs, à dérivation plus courte, à préférer des ouvrages déchargeurs à plus faible débit (à peu près la moitié du débit d'équipement) mais à ouverture rapide, en un temps comparable à celui de la fermeture des turbines ( 10 à 20 secondes).

Ces ouvrages déchargeurs étaient très coûteux. Ils posaient des problèmes sérieux, aussi bien pour la manœuvre des vannes que pour la dissipation de l'énergie de l'eau. On craignait, lors des premières réalisations en vannes rapides, que leur fonctionnement ne soit pas absolument fiable. D'où l'idée de remplacer plus ou moins complètement cet ouvrage spécial par une mancuvre permettant de faire passer le débit par les turbines elles-mêmes, le groupe étant découplé du réseau. C'est la marche en déchargeur.

$\mathrm{Ce}$ fonctionnement, assez facilement réalisé sur des machines moyennes (réalisation EDF de Palaminy), s'est d'abord révélé décevant sur les plus grosses. L'existence, pour les raisons exposées plus haut, de vannes aval, a ouvert une voie nouvelle de recherche: réaliser ce fonctionnement en réduisant le plus possible la chute sur la roue (ce qui conduit à placer la turbine en position d'emballement à débit maximal), et en contrôlant ce débit par la vanne aval. Les premiers essais réalisés industriellement en profitant de la vanne banale du Logis-Neuf ont été assez concluants pour qu'on adopte ce fonctionnement dès Beauchastel, mais, cette fois, comme modalité d'exploitation puisque toutes les machines possédaient une vanne à demeure.

L'ouvrage déchargeur traditionnellement accolé à l'usine était maintenu, faute de disposer encore du moyen permettant de passer de la marche en charge à la marche en déchargeur en une variation monotone du débit. Cependant, dès Beauchastel, on approchait de ce résultat en provoquant la descente des vannes (encore lentes) dès le déclenchement, et en plaçant le groupe, en attendant la prise de contrôle de débit par les vannes, dans une position intermédiaire de "marche en déchargeur déconjuguée sans vanne", pas très confortable, mais acceptable pour une courte période. Pour des machines qui, en charge maximale, turbinaient $350 \mathrm{~m}^{3} / \mathrm{s}$, on passait par une situation intermédiaire de l'ordre de $100 \mathrm{~m}^{3} / \mathrm{s}$ pendant 3 minutes environ puis, la vanne prenant le contrôle, à une marche en déchargeur définitive très calme à $200 \mathrm{~m}^{3} / \mathrm{s}$ environ.

Dès cette époque, on avait donc réalisé ainsi, avec les 6 groupes et presque gratuitement (aucun équipement lourd; seulement quelques relais dans les schémas) un déchargeur rapide de $600 \mathrm{~m}^{3} / \mathrm{s}$ relayé par un déchargeur lent de $1200 \mathrm{~m}^{3} / \mathrm{s}$. Même en prenant par prudence, comme on le fait généralement, l'hypothèse d'un groupe fonctionnant mal pendant le transitoire et passant à l'arrêt, ce qui ramène les valeurs précédentes à 500 et $1000 \mathrm{~m}^{3} / \mathrm{s}$, on voit que cette étape réalisait, sur turbines Kaplan, une marche en déchargeur rapide de $24 \%$ du débil d'équipement, et une marche en déchargeur lente de $48 \%$ de ce même débit.

C'est alors que, pour les raisons exposées plus loin, nous décidions d'équiper les usines suivantes, sauf Bourg-lès-Valence ${ }^{(2)}$, de turbines bulbes, qui, comme on pourrait le démontrer, en raison même du fait qu'elles sont plus "poussées", ont un débit d'emballement relativement plus fort que les Kaplan équivalentes, et de ce fait une aptitude, toutes conditions semblables par ailleurs, et toujours bien sûr en technique "marche

(2) Pierre-Bénite venait d'être décidé. On souhaitait, par prudence, ne pas engager la tranche de taille supérieure sans être assuré de pouvoir tenir compte des premiers résultats de Pierre-Bénite pour retoucher éventuellement certains points de construction. C'est pourquoi la série des $30 / 35 \mathrm{MW}$ n'a été lancée qu'à Vallabrègues. 
en déchargeur contrôlée par vanne aval', à un rapport débit en déchargeur/débit nominal plus fort que la turbine Kaplan équivalente. D'emblée, on adopta un objectif de débit déchargé d'au moins $70 \%$ du débit d'équipement, avec en outre la double volonté d'établir ce débit, à partir de la marche en charge, par une décroissance monotone, et, l'incident passé, de repasser également en marche en charge par une croissance monotone du débit. C'est ainsi qu'est née la "vanne aval rapide", perfectionnement pratiquement gratuit de la vanne gravitaire traditionnelle, qui permet d'obtenir ce double résultat, et qui fut appliquée à toutes les chutes. Des perfectionnements de la régulation permettent même, depuis Vallabrègues, de rétablir strictement en déchar. geur le débit qui existait avant le déclenchement toutes les fois (fréquentes) que ce débit ne dépasse pas $70 \%$ du débit maximal. Une mise au point du recouplage du groupe directement par la vanne, sans passer par la "marche à vide" a permis d'atteindre tous les objectifs recherchés.

\section{Les groupes bulbes}

On a beaucoup parlé des groupes bulbes, spécialement de ceux du Rhône [4] et des communications de constructeurs en reparleront durant cette session. Il suffit de rappeler que l'équipement du Rhône a joué, en ce domaine, un rôle important :

- d'abord en enclenchant avec l'équipement de PierreBénite (choix déterminé vers 1962) un processus irréversible des gros équipements de basse chute selon cette technique, choix qui repoussait significativement, par une économie globale de $20 \%$ sur l'usine, les limites de rentabilité des aménagements;

- en permettant de poursuivre l'aménagement mixte du Rhône à des époques où les perspectives étaient peu favorables aux équipements hydrauliques;

- puis en prolongeant ce mouvement, pour rentabiliser les "derniers groupes", les plus marginaux de chaque aménagement, par l'introduction de gros groupes bulbes très simplifiés, à distributeur fixe et roue hélice à pales non orientables, de façon à maintenir l'homogénéité des débits d'équipement aux périodes économiques les moins favorables à l'hydraulique (Sauveterre, conçu vers 1969, puis Caderousse, en "usine mixte", conçue vers 1971).

Ces grandes réalisations continues ont certainement favorisé la diffusion de cette technique, qui représente actuellement un parc mondial d'environ $5000 \mathrm{MW}$ où la construction française vient largement en tête (environ la moitié) devant 1'URSS (environ un quart), puis les constructions d'origine suisse et allemande, souvent associées, avec, notamment pour les groupes du Danube, une participation importante de constructeurs autrichiens, puis la plupart des autres constructeurs européens, notamment suédois et italiens.

Sur l'ensemble de ce parc, les groupes bulbes du Rhône totalisent environ $1350 \mathrm{MW}$ en 44 groupes, les plus petits $(18 \mathrm{MW})$ à Vaugris sous $6 \mathrm{~m}$ de chute, les plus gros ( $45 \mathrm{MW})$ sur le Haut Rhône, à Chautagne et à Belley, sous $18 \mathrm{~m}$ de chute.

A ce parc s'ajoute un ensemble de microcentrales de techniques diverses (plus de 10 machines installées ou en projet) pour la récupération, en pied de barrages, des débits réservés dans les portions du Rhône courtcircuitées [6].

L'ensemble du parc de grosses machines se caractérise par une certaine homogénéité des tailles (de $6,10 \mathrm{~m}$ à $6,40 \mathrm{~m}$ de diamètre en groupes règlables; $6,90 \mathrm{~m}$ en groupes sans règlage) et des débits unitaires (350 à $400 \mathrm{~m}^{3} / \mathrm{s}$ ), résultant d'études d'optimisation plusieurs fois renouvelées, et par une évolution des caractéristiques de fonctionnement (coefficient de vitesse, débit spécifique, etc.) adaptées à la fois à l'évolution des données économiques générales et des données d'installation (nature des fondations par exemple). On retrouverait ici l'imbrication des données économiques, des impératifs généraux d'aménagements, et des évolutions technologiques propres à chaque type d'équipement qui ont été mises en évidence plus haut par l'exemple des vannes. Cette remarque mériterait évidemment un développement, qui pourra être donné dans des communications ultérieures.

\section{Marche en éclusee et automatisation des aménagements}

Le Rhône est, à notre connaissance, le seul ensemble hydraulique constitué d'une chaîne d'aménagements mixtes de 19 chutes (12 en aval de Lyon, 7 en amont), entièrement automatisé. Cette automatisation, réalisée en étroite collaboration avec EDF n'a nullement été motivée par un souci de modernisme, ni principalement pour des raisons de personnel (encore que la suppression de tâches répétitives comportant par contre mais très rarement des interventions fort difficiles présente un intérêt humain certain), mais pour résoudre au mieux les importants problèmes posés par les divers modes de gestion de ces ouvrages en chaîne (marches au fil de l'eau; marches en éclusée; marches en déversement; fonctionnements en crue; traitement des perturbations techniques - pannes, déclenchements. . . - et hydrauliques - crues subites d'affluents rapides par exemple -).

Cet ensemble, réalisé sous une forme très décentralisée, apportant le maximum de sécurité mais avec des supervisions permettant toutes les politiques d'enchaînement, voire des interventions globales en cas de besoin inopiné des réseaux, a nécessité une analyse extrêmement détaillée de l'exploitation, et une étude hydraulique très complète des régimes permanent et transitoire.

Le fait déterminant de cet équipement a été le "fonctionnement en éclusées" qui permet, pendant les 4 heures que peut durer une demande énergétique, de mobiliser une puissance équivalente à celle d'une tranche thermique moderne qui vient s'ajouter à la puissance moyenne totale de l'aménagement.

Là encore, on pourrait montrer de quelle façon la réponse à des objectifs de valorisation de l'énergie, à l'origine très modestes, a conduit à développer un équipement très moderne qui, à son tour, a permis de réaliser de nouveaux objectifs tels que :

- l'intervention très rapide de puissance pour équilibrer le réseau de transport en cas de défaillance d'autres sources ;

- l'intervention "en chaîne" accroissant la participation d'une usine de tête au réglage national. 
Ces objectifs, il y a une ou deux décennies, n'auraient jamais été confiés à des usines hydrauliques de basse chute qui étaient uniquement réservées à la base du diagramme de puissance. Il n'est plus rare maintenant de voir un groupe du Rhône démarrer et s'arrêter trois fois par jour, alors qu'il y a vingt ans ce groupe n'aurait été mis en route et arrêté que trois fois dans l'année.

Il est frappant de constater que les machines et la plupart des organes soutiennent très bien cet accroissement de tâches, avec des espacements de gros entretiens qui, pour des raison diverses, deviennent de plus en plus longs (une ou plusieurs décennies).

\section{Equipement des écluses}

Toutes les écluses du Rhône sont à grand gabarit, avec sas de $12 \mathrm{~m}$ de largeur et $195 \mathrm{~m}$ de longueur, et mouillage garanti d'au moins $3 \mathrm{~m}$. Beaucoup de ces équipements, et les études correspondantes, ont été récemment présentés à la SHF [5]. Rappelons seulement, là encore, une évolution vers un équipement de plus en plus discret et de plus en plus fiable (portes roulantes dans des garages; pare-chocs à grosse énergie) qui tient compte de l'obligation d'un service permanent, avec la présence d'une écluse unique à chaque aménagement dont les équipements principaux, qui à l'origine étaient doublés, sont devenus uniques depuis plus de 10 ans par mesure d'économie.

\section{Equipement des barrages}

Les équipements de barrages ( 21 barrages principaux, dont 20 barrages mobiles en rivière) ont suivi les mêmes évolutions que ceux des usines :

- optimisation économique, qui, des premiers barrages en vannes-wagon à crochets a fait passer, (depuis Bourg-lès-Valence, mis en service en 1968) à des vannessegment, moins chargées de mécanique, dont l'évolution de prix a été moins sensible que celui de la charpente; - diminution des volumes et discrétion des ouvrages, exigeant de nouvelles cinématiques, et profitant d'autre part des progrès importants du génie civil.

L'avancement des technologies, le souci esthétique, ont même conduit à projeter pour l'aménagement de
Brégnier-Cordon des vannes segment inversées dont la mécanique sera très peu visible.

\section{Conclusion}

Il est difficile dans un seul exposé de présenter, avec tous les développements techniques que pourrait souhaiter l'auditoire de la Société Hydrotechnique de France, la totalité des études, des évolutions et des innovations effectuées à l'occasion d'un grand aménagement de fleuve tel que celui du Rhône. Nous espérons cependant que les quelques exemples présentés donneront une idée de l'expérience très étendue que peut acquérir un maitre d'œuvre ayant conçu et réalisé de longue haleine une cuvre homogène. Il acquiert ainsi une vue d'ensemble, dépassant la simple juxtaposition de divers équipements; il atteint une connaissance complète de leurs interconnexions, et peut intégrer à l'évolution des techniques les résultats de son expérience continue d'équipement et d'exploitation.

\section{Références bibliographiques}

[1] ROBERT A. - L'équipement hydro-électrique et mécanique de la Centrale, numéro hors série de la Houille Blanche.

[2] MM. CAZENAVE, MARTIN-RASCALON, CHEVALIERL'HERMITE, CAMPMAS, MEGNINT, ARNOUD-CANTE. 5 communications sur la marche en déchargeur, SHF (session des 16 et 17 novembre 1967), numéro spécial Houille Blanche 2-3,1968.

[3] GEMAEHLING Cl. - Quelques aspects des études hydrauliques des aménagements du Bas-Rhône SHF (Session du 16 novembre 1962), numéro spécial Houille Blanche $B-1962$

[4] MM. COTILLON, ANDRE, BESLIN, CASACCI - 4 communications sur les groupes bulbes en rivière, SHF (Session des 16 et 17 novembre 1972), numéro houille Blanche 2-3, 1973 .

[5] PINATEL R. - Les écluses d'une voie à grand gabarit : Le Rhône.

MM. DUBOIS, CORNIER, MAMET, CORBIC. - Problèmes des chocs de bateaux sur les ouvrages, SHF (Session des 19 et 20 novembre 1980), numéro Houille Blanche 2-3, 1981 (hydrotecnique et Navigation Intérieure).

[6] ROUYER J.P. - LE PLOMB C. - Récupération d'énergie hydraulique : petites installations d'énergie associées à de grands projets hydrauliques (Session du 18 et 19 mars 1981. La Houille Blanche n. $4-5,1981$ ).

\section{Discussion}

Président : M. DEVOISSELLE

M. le Président. - Je remercie MM. SAVEY et CAZENAVE de leur exposé. Nous venons d'assister à la présentation d'un aménagement à buts multiples. Je pense qu'il a fallu l'obstination des dirigeants de la CNR pendant près d'un demi-siècle pour mener à bien ces travaux au milieu de conjonctures qui ont été, du point de vue politique et économique, parfois très difficiles.
Si quelqu'un veut un jour écrire I'histoire des progrès de la technique et de la technologie, je lui conseille de se promener sur le Rhône : il verra les différentes étapes qui ont été franchies; et, faisant cette promenade, il pourra écrire une histoire très intéressante. $M$. CAZENAVE, ne manquera pas de lui fournir les informations les plus précises. 


\section{Abstract \\ Integrated development of a large river: the Rhône \\ B. Development of electro-mechanical equipments}

It took half a century to develop the Rhone river between Génissiat and Loyettes. There have beer many changes vis-àvis the initial objectives (economic optimisation, operating safety and flexibility, easing of maintenance procedures).

Low heads ( 5.70 to 21 meters) have been harnessed. In addition, operating constraints relating to energy generation, navigation had to be complied with and substantial variations in flows and heads allowed for. The plants are equipped with vertical axial turbines for the alder sets and horizontal for the new ones.

Technological improvements and operating experience have led to the major changes summarized below.

1) Flow and head: increase in the flow handled by plants and possibility of turbining low heads.

2) Safety valves: use of downstream cuts; in 1958, use of one valve per set, an acceptable measure for up to 6 or 8 sets gravity flow valves and single stroke servomotors; Optimization of the position of valves.

3) Discharge facilities: the use of the circuit of the turbine itself has been studied for rapid discharge, initially tor the
Kaplan turbines and later for the bulb sets. It is now possible, following this method, to draw on a flow of up to $70 \%$ of maximum flow.

4) Bulb generating sets: the harnessing of the Rhône has played a major role in this field. France accounts for about half of the World park of $5000 \mathrm{MW}$; the sets on the Rhone alone total $1350 \mathrm{MW}$ for 44 sets (18 MW under 6 metre heads to $45 \mathrm{MW}$ under $18 \mathrm{~m}$ ).

5) Automation of developments: the fully automated series of mixed projects harnessing 19 heads on the Rhône, is a World first. It allows lock operation to be performed. The ultramodern equipment allows rapid coupling to the system.

6) Locks: the large locks on $(12 \times 195 \mathrm{~m})$ the Rhône have led to the development of increasingly reliable units of equipment, necessitated by the fact that each lock is in permanent service.

7) Harnessing of dams: 21 main dams have been built including twenty mobile dams on the rivers. Major progress has been made in construction, especially as regards gates. 